\title{
ON Some Desired Properties of DATA AUGMENTATION BY ILLUMINATION SIMULATION FOR COLOR CONSTANCY
}

\author{
Nikola Banić ${ }^{1}$, Karlo Koščević2 , Marko Subašić2 ${ }^{2}$ and Sven Lončarić2 \\ ${ }^{1}$ Gideon Brothers, 10000 Zagreb, Croatia \\ ${ }^{2}$ Faculty of Electrical Engineering and Computing, \\ University of Zagreb, 10000 Zagreb, Croatia
}

\begin{abstract}
Computational color constancy is used in almost all digital cameras to reduce the influence of scene illumination on object colors. Many of the highly accurate published illumination estimation methods use deep learning, which relies on large amounts of images with known ground-truth illuminations. Since the size of the appropriate publicly available training datasets is relatively small, data augmentation is often used also by simulating the appearance of a given image under another illumination. Still, there are practically no reports on any desired properties of such simulated images or on the limits of their usability. In this paper, several experiments for determining some of these properties are proposed and conducted by comparing the behavior of the simplest illumination estimation methods on images of the same scenes obtained under real illuminations and images obtained through data augmentation. The experimental results are presented and discussed.
\end{abstract}

\section{KEYWORDS}

Color constancy, data augmentation, illumination estimation, image enhancement, white balancing.

\section{INTRODUCTION}

The feature of the human visual system (HVS) that allows for object color recognition regardless of the scene illumination is known as color constancy [1]. Computational color constancy is present in the image processing pipelines of almost all digital cameras. Its most challenging task is the illumination estimation and for that the following image $\mathbf{f}$ formation model, which also includes the Lambertian assumption, is most commonly used

$$
\mathbf{f}_{\mathbf{c}}(\mathbf{x})=\int_{\omega} \mathbf{I}(\lambda, \mathbf{x}) \mathbf{R}(\lambda, \mathbf{x}) \boldsymbol{\rho}_{\mathbf{c}}(\lambda) \mathbf{d} \lambda
$$

where $\mathrm{c}$ is a color channel, $\mathbf{x}$ is a given image pixel, $\lambda$ is the wavelength of the light, $\omega$ is the visible spectrum, $\mathrm{I}(\lambda, \mathbf{x})$ is the spectral distribution of the light source, $\mathrm{R}(\lambda, \mathbf{x})$ is the surface reflectance, and $\rho_{c}(\lambda)$ is the camera sensitivity of the c-th color channel. Since uniform illumination is usually assumed, $\mathbf{x}$ is removed from $\mathrm{I}(\lambda, \mathbf{x})$ so the observed light source color is then 


$$
\mathbf{e}=\left(\begin{array}{l}
\mathbf{e}_{\mathrm{R}} \\
\mathbf{e}_{\mathrm{G}} \\
\mathbf{e}_{\mathrm{B}}
\end{array}\right)=\int_{\omega} \mathbf{I}(\boldsymbol{\lambda}) \boldsymbol{\rho}(\boldsymbol{\lambda}) \mathbf{d} \boldsymbol{\lambda} .
$$

Already the direction of $\mathbf{e}$ is sufficient for a successful color correction [2]. With only image pixel values $\mathbf{f}$ being given and both $\mathrm{I}(\lambda)$ and $\boldsymbol{\rho}(\lambda)$ being unknown, estimating $\mathbf{e}$ is an ill-posed problem, which requires additional assumptions. This has led to development of numerous methods and recently, there is a growing trend of proposing various learning-based methods. Many of these methods rely on large amounts of data for performing successful learning and since the publicly available training datasets are relatively small, some form of data augmentation is usually used. One of the augmentation techniques involves simulating as if an image was taken under another illumination by performing simple multiplications of the color channel values. Nevertheless, there is practically no report on the properties that the images obtained through such data augmentation should have. Therefore, the goal of this paper is to propose and perform several simple experiments that can numerically show at least some of the mentioned properties.

The paper is structured as follows: some of the related work is described in Section 3, the motivation for checking for some of the properties that the images obtained through data augmentation by illumination simulation should have is given in Section 3, the experimental setup is proposed in Section 4, the experimental results are presented and discussed in Section 5, and, finally, Section 6 concludes the paper.

\section{RELATED WORK}

Based on the kind of the assumptions that they use, illumination estimation methods can roughly be divided into two groups. The first group are low-level statistic-based methods such as Whitepatch [3], [4], its improvements [5], [6], [7], Gray-world [8], Shades-of-Gray [9], Gray-Edge (1 and $2^{\text {nd }}$ order) [10], using bright and dark colors [11], etc.

On the other hand the second group consists of learning-based methods such as gamut mapping (pixel, edge, and intersection based) [12], using high-level visual information [13], natural image statistics [14], Bayesian learning [15], spatio-spectral learning (maximum likelihood estimate, and with gen. prior) [16], simplifying the illumination solution space [17], [18], [19], using color/edge moments [20], using regression trees with simple features from color distribution statistics [21], performing various kinds of spatial localizations [22], [23], using convolutional neural networks [24], [25], [26], [27] and genetic algorithms [28], modelling colour constancy by using the overlapping asymmetric Gaussian kernels with surround pixel contrast based sizes [29], finding paths for the longest dichromatic line produces by specular pixels [30], detecting gray pixels with specific illuminant-invariant measures in logarithmic space [31], channel-wise pooling the responses of double-opponency cells in LMS color space [32], and numerous other. Low-level statistics-based method rely on simple image statistics and therefore, they are fast, computationally cheap, and suitable for hardware implementation. Learning-based methods are more complex and computationally expensive, but they also have the highest accuracy, which has recently often been achieved through various deep learning approaches. However, to achieve high accuracy, methods based on deep learning usually require substantial amount of training data. Since this condition may not always be met, it is not uncommon to perform data augmentation. 


\section{Motivation}

\subsection{Augmentation through illumination simulation}

One of the techniques of data augmentation used for computational color constancy methods' training is to multiply the image color channels in order to simulate another illumination in rough accordance with Eq. (1). Let $\mathbf{f}^{(\mathbf{e})}$ be an image taken under the observed light source $\mathbf{e}$. If $\hat{\mathbf{f}}^{\left(\mathbf{e}^{\prime}\right)}$ is the simulation of $\mathbf{f}^{(\mathbf{e})}$ being taken under the observed light source $\mathbf{e}^{\prime}$, the channel $\mathrm{c}$ value of a pixel at location $\mathbf{x}$ is then calculated as

$$
\hat{\mathbf{f}}_{\mathbf{c}}^{\left(\mathbf{e}^{\prime}\right)}(\mathbf{x})=\frac{\mathbf{e}_{\mathbf{c}}^{\prime}}{\mathbf{e}_{\mathbf{c}}} \mathbf{f}_{\mathbf{c}}^{(\mathbf{e})}(\mathbf{x})
$$

For example in [36] and [37] this is done by taking existing image patches and then multiplying the color values of their pixels and the color values of their corresponding ground-truth illuminations by random factors so that $\frac{e_{c}^{\prime}}{e_{c}} \in[0.8,1.2]$ for every channel $c$. This is a practical simplification, which is also often used for color correction and known as von Kries diagonal model [38]. Since Eq. (3) is a vast simplification of Eq. (1) that does not include inter-channel connections, it should have no effect on the error of moment-based methods such as Gray-world or its generalization Shades-of-Gray if the effects of intensity rounding are ignored. The illumination estimation performed by the Shades-of-Gray method is

$$
\left(\frac{\int(\mathbf{f}(\mathbf{x}))^{\mathbf{p}} \mathbf{d x}}{\int \mathbf{d} \mathbf{x}}\right)^{\frac{1}{\mathbf{p}}}=\mathbf{e}
$$

The Gray-world method is just a special case of the Shades-of-Gray method with $p=1$. The error of these methods obtained on the augmented images should by definition remain the same except for the rounding errors. However, these methods are some of the fundamental methods of color constancy and they are at the core of many successful methods mentioned in the previous section. Therefore, it can be argued that the images obtained through data augmentation by illumination simulation should behave similarly to real images of the same scene taken under different illuminations. In other words, while the difference between $\mathbf{e}$ and $\mathbf{e}^{\prime}$ has almost no effect on the errors that occur by applying Eq. (4) to $\mathbf{f}^{(\mathbf{e})}$ and $\hat{\mathbf{f}}^{\left(\mathbf{e}^{\prime}\right)}$, it does have an effect when they are applied to $\mathbf{f}^{(\mathbf{e})}$ and $\mathbf{f}^{\left(\mathbf{e}^{\prime}\right)}$. Measuring the extent of that effect by appropriate experiments should also give more insight into how real augmented images should behave. An example of such behavior and how these experiments could be performed are given in the next subsection. 


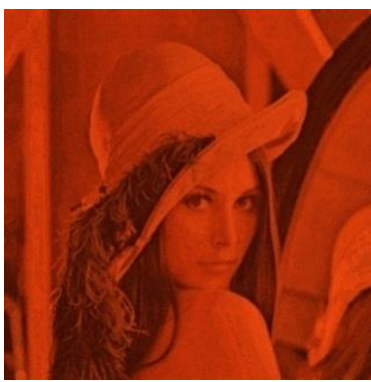

(a)

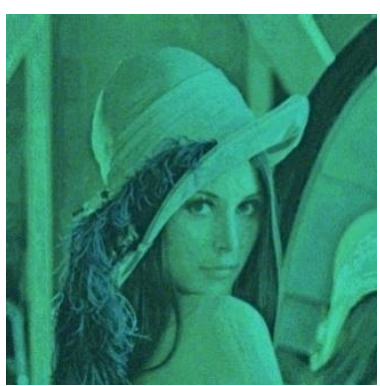

(b)

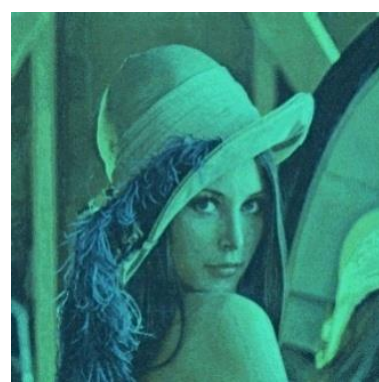

(c)

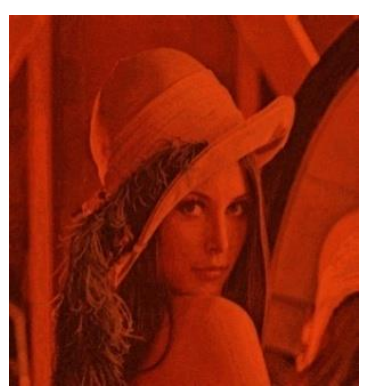

(d)

Figure 1. Examples of data augmentation by illumination simulation on images generated by the CroP dataset generator [33] and tone mapped by the Flash tone mapping operator [34] for display purposes: (a) the linear raw image of the printed photography taken under the red light, (b) the simulation of taking the image under the white light by applying Eq. (3) to the previous image, (c) the linear raw image of the printed photography taken under the white light, and (d) the simulation of taking the image under the red light by applying Eq. (3) to the previous image. The reproduction angular error [35]obtained by Grayworld for the images are $7.01^{\circ} 7.06^{\circ}, 4.63^{\circ}$, and $4.62^{\circ}$ respectively, which means that despite a change in the appearance the simulation had little effect on the moment-based Gray-World method.

\subsection{The CroP dataset generator}

In order to perform such experiments, it should be possible to obtain images of the same scene under various illuminations. While some datasets such as the ones in [39] and [40] have images of same scenes under various illuminations, the number of the illuminations is limited and the images are not available in the raw form that contains linear intensities expected by Eq. (1).

A suitable solution for this problem would be to use the CroP dataset generator [33]. CroP allows for generating highly realistic simulations of raw images that follow the linear model used by Eq. (1). It simulates photographing a paper with printed images under one of 707 different illuminations. The paper is fixed and the colors are also somewhat restricted, which also limits the $\mathrm{R}(\mathbf{x}, \lambda)$ from Eq. (1) to the one of paper. Nevertheless, even with these constraints, it still represents a valuable tool because exactly the same scene can be obtained under different illuminations without any need of image registration and with all the effects of $R(\mathbf{x}, \lambda)$. The 707 illuminations were chosen to cover most of the chromaticity plane with a higher density around the chromaticities of the colors of the ideal black body. In this paper CroP is used to simulate taking of images as if the Canon EOS 6D Mark II camera was being used. The use of CroP can be considered innovative when compared to the commonly used light simulation techniques. The main advantages of using CroP are the high number of supported illuminations and the realistic rendering that includes the physical properties of the paper, while the main disadvantages are having the paper as the only currently supported material and being restricted to a flat plane, which are also the main deficiencies that can be observed when using CroP in the described way.

A simple way to demonstrate the difference between using Eq. (3) and CroP is to apply e.g. the Gray-world method to images created by using both these approaches and then to compare the obtained results by measuring the error of the estimations. The reproduction angular error [35] was shown to be the most appropriate way to measure the errors of illumination estimation methods so for this reason it is used in the rest of the paper. It is defined as "the angle between the image RGB of a white surface when the actual and estimated illuminations are divided out" [35].

Figure 1 shows an example of images generated by CroP and then modified by Eq (4). First an image taken under the red light was created and then it was modified by Eq. (4) to appear as if it was taken under the white light. The reproduction angular error obtained by the Gray-world 
method was roughly the same around $7^{\circ}$ for both images due to its momentum-based nature, i.e. simulating the white light on the image obtained under the red light had no effect on the Grayworld's estimation error. When the roles of the red and white light were reversed, the errors on the next two new images were again practically the same around $4.6^{\circ}$, but they obviously differed from the errors obtained on the first two images. Namely, due to its spectral characteristics the real red illumination on the first image hides many details in the green and blue channel, while the simulated red in the last image only slightly changes, but it still retains the information in these channels and thus the obtained errors also differ. Already this example shows how much images obtained by simple data augmentation can differ from the realistic images they are supposed to simulate.

\section{Proposed Experiments}

There are several questions that may be interesting in terms of the differences between the real images and the ones obtained through data augmentation by illumination simulation and they are centered around the behavior of colors under various illuminations.

First, as shown by the example in Figure 1, the images obtained through illumination simulation by using Eq. (3) will give a very similar error when moment-based illumination estimation methods are applied to them. However, this example has also shown that in the case of images taken under real illuminations the estimation error also depends on the illumination color. Therefore, the first question is to what extent can the illumination color influence the estimation error of moment-based methods?

Second, while there is a significant variation in estimation errors when the illumination colors differ significantly as shown in Figure 1, for similar illuminations the difference may not be statistically significant. Therefore, the second question is how much does the illumination color have to change in order to also significantly change the estimation error?

Third, while the illumination color can be arbitrary when artificial light sources are used, in practice the digital cameras mostly focus only on a restricted set of illuminations, usually the ones whose chromaticities are close to the ones of the black body radiation colors [28]. Therefore, when looking for an answer to the first two questions, it would be useful to give it individually for the case when a large variety of illuminations, e.g. all 707 illuminations from CroP, are taken into account, but also for the case when only the illuminations close to the common real-world illuminations are taken into account.

\section{EXPERIMENTAL RESUlts}

\subsection{Experimental Setup}

The images used for the experiments are the 14 well-known color images from the Volume 3 of the SIPI Image Database [41]. For the moment-based methods the Gray-world and the Shades-ofGray were used with $\boldsymbol{p}=\mathbf{4}$ for the latter. The error metric is the already mentioned reproduction angular error [35] defined formally as

$$
\mathbf{d}(\mathbf{g}, \mathbf{e})=\cos ^{-1}\left(\frac{\frac{\mathbf{g}_{\mathrm{R}}}{\mathbf{e}_{\mathrm{R}}}+\frac{\mathbf{g}_{\mathrm{G}}}{\mathbf{e}_{\mathrm{G}}}+\frac{\mathbf{g}_{\mathrm{B}}}{\mathbf{e}_{\mathrm{B}}}}{\sqrt{3} \cdot\left\|\left(\frac{\mathbf{g}_{\mathrm{R}}}{\mathbf{e}_{\mathrm{R}}}, \frac{\mathbf{g}_{\mathrm{G}}}{\mathbf{e}_{\mathrm{G}}}, \frac{\mathbf{g}_{\mathrm{B}}}{\mathbf{e}_{\mathrm{B}}}\right)\right\|}\right)
$$


where $\boldsymbol{g}$ is the ground-truth observed illumination and $\boldsymbol{e}$ is the illumination estimation. To observe the effect of various illuminations on the reproduction angular error obtained by applying the Gray-world and Shades-of-Gray methods to the test images, the images' appearance under the given illuminations in the raw linear form was realistically simulated by using the previously described CroP dataset generator.

The CroP database generator allows for 707 different illuminations evenly spread across the chromaticity plane. One of the currently largest publicly available single camera color constancy benchmark dataset called Cube+ [42] has 1707 different real-world ground-truth illuminations and it was created by using a Canon EOS 550D camera. When for each of the Cube+ groundtruth illuminations the closest illumination available in the CroP is taken, the result is a set of 76 different illuminations and these will be used for the experiments that examine the influence of real-world illuminations.

\subsection{Influence of illumination color on estimation error}

To see to what extent does the illumination color influence the estimation error of moment-based methods, for each of the 707 illuminations available in the CroP dataset generator a small dataset was created based on the SIPI Image Database color images, the Gray-world and the Shades-ofGray methods were applied to them, and for each of these methods the mean reproduction angular error was calculated. This resulted in 707 such errors for each method, one per each illumination, and after the outliers were dropped out, the obtained distribution of the reproduction angular errors was as shown in Figure 2 and Figure 3. In both cases the mean angular error spans a range of over two degrees, which shows also effectively shows the extant of the influence of the illumination color on the estimation error. Additionally, the mean angular errors closer to the center of both of the ranges occur more often than the errors closer to the range limits.

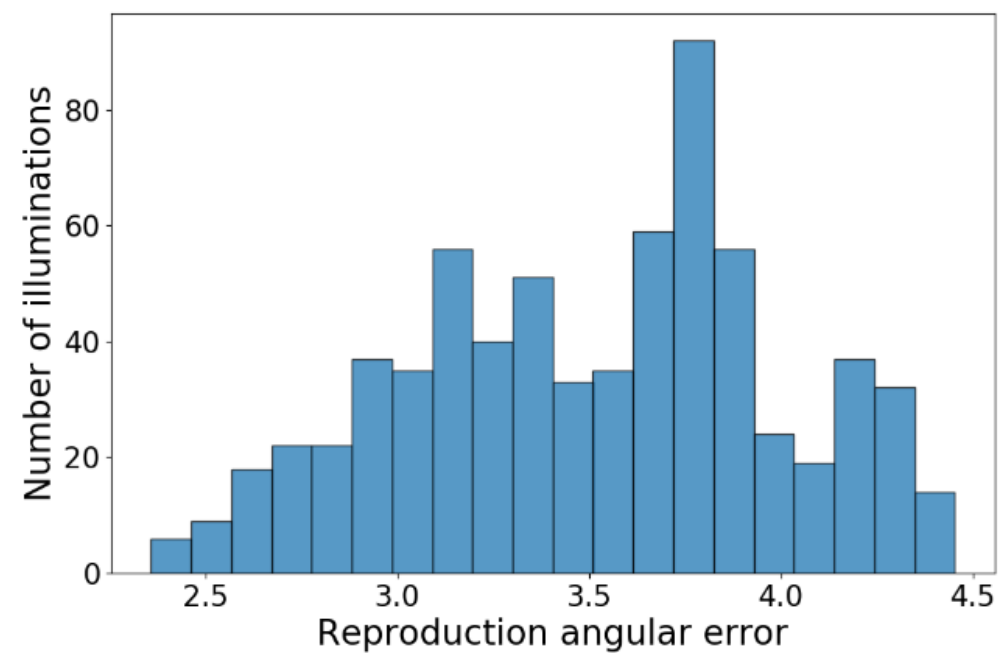

Figure 2. Distribution of the mean reproduction angular errors obtained by applying the Gray-world method to the test images generated by CroP. Each mean was obtained for all test images generated by CroP by fixing the illumination to one of the 707 illuminations that are available in CroP. 


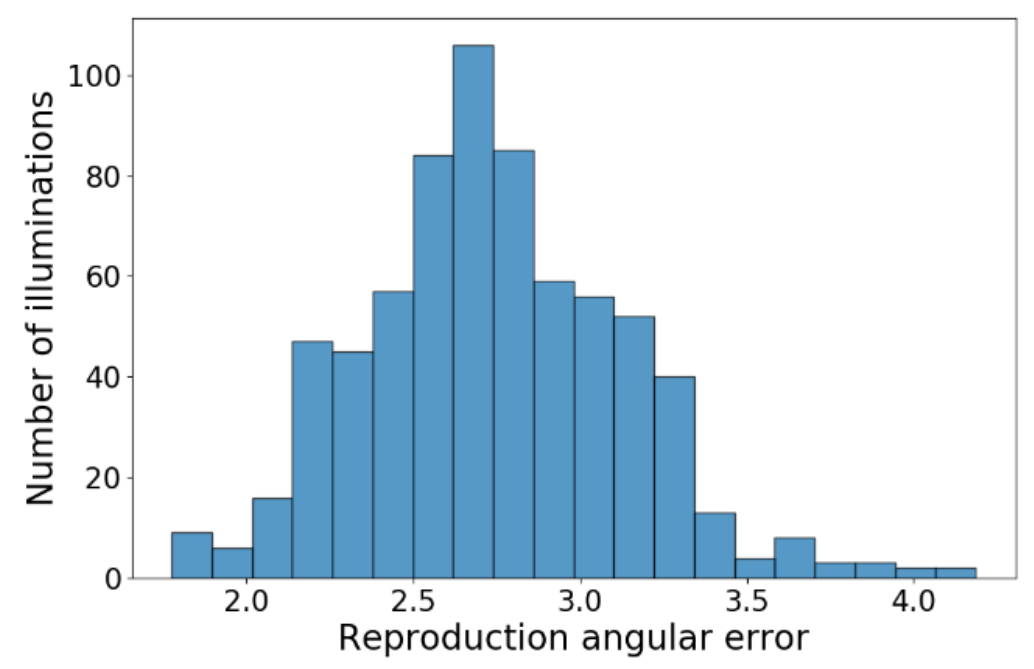

Figure 3. Distribution of the mean reproduction angular errors obtained by applying the Shades-of-Gray method to the test images generated by CroP. Each mean was obtained for all test images generated by CroP by fixing the illumination to one of the 707 illuminations that are available in CroP.

When the same experiment is repeated for the 76 of the CroP illuminations that more often occur in scenes of real-world images, the results shown in Figure 4 and Figure 5 show that the error spans a range of over half a degree. As expected, this is less than in the previous case due to less variation, but still significant.

These results show how the scene information is changed depending on the illumination and some good data augmentation should also have such effect.

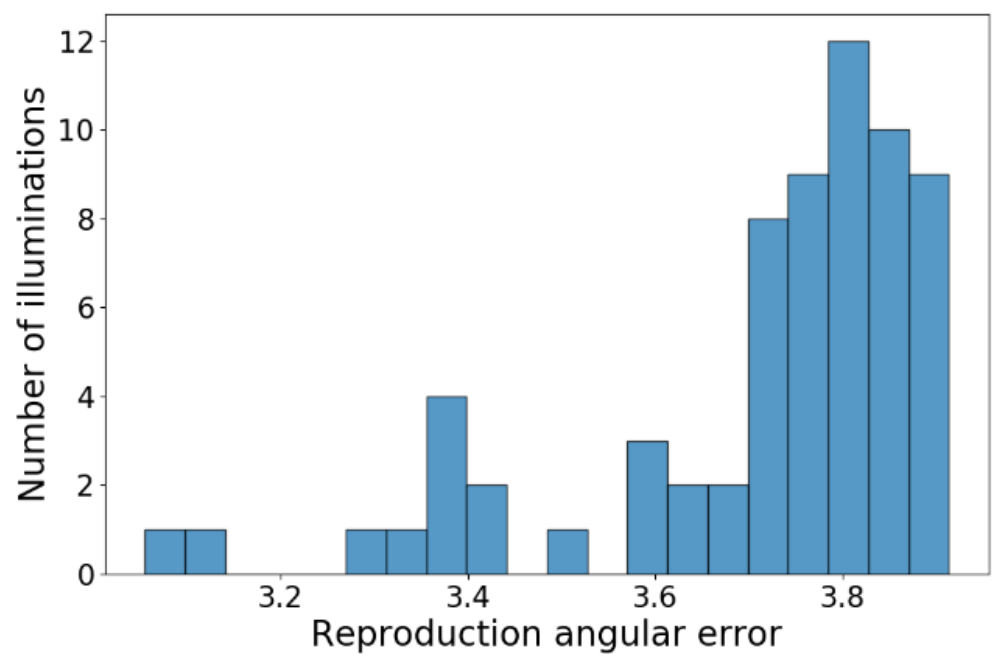

Figure 4. Distribution of the mean reproduction angular errors obtained by applying the Gray-world method to the test images generated by CroP. Each mean was obtained for all test images generated by Crop by fixing the illumination to one of the 76 ones commonly seen in real-world. 


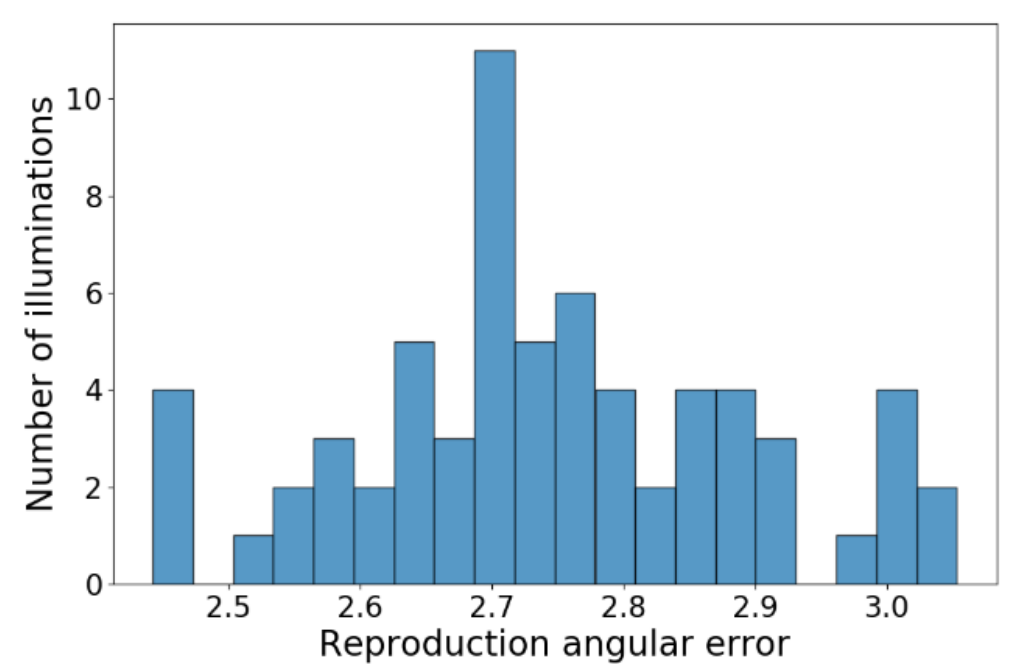

Figure 5. Distribution of the mean reproduction angular errors obtained by applying the Shades-of-Gray method to the test images generated by CroP. Each mean was obtained for all test images generated by Crop by fixing the illumination to one of the 76 ones commonly seen in the real-world.

\subsection{Minimum required change}

To check the minimum change of the illumination color required for a statistically significant change in the illumination estimation errors, for each of the 707 illuminations available in the CroP dataset the respective datasets were generated out of the SIPI Image Database images and the reproduction angular errors were calculated for each of them. Then for each two pairs of perimage angles the non-parametric Wilcoxon signed-rank test [43] was used since the angular errors are not normally distributed [44]. The usually used significance level is $\alpha=0.05$, but since numerous illumination pair comparisons are supposed to be performed, this opens the problem of multiple comparisons [45]. One way to counter it would be to apply the Bonferroni correction [46], but if all $m=\frac{707 \cdot 706}{2}$ pairs are to be compared, then the significance level has to be set to $\alpha=\frac{0.05}{\mathrm{~m}} \approx 2 \cdot 10^{-7}$, which results in a too high conservatism that rejects any null hypothesis that the two angle samples have equal means. It should be mentioned that this is despite the Wilcoxon's test reduced statistical power due to it being non-parametric. By using $\alpha=$ 0.001 , only around $0.18 \%$ of the hypotheses are not rejected, while for $\alpha=0.01$ this raises to around $3.4 \%$. Nevertheless, due to the mentioned multiple comparison problem the last two mentioned results are not statistically valid because they allow for the random sampling error to have a too big influence.

After trying to create plots that would e.g. show the mean angle between illuminations obtained for $p$-values under a certain $\alpha$ or vice versa, it was concluded that the influence of the random sampling noise was too large. In short, the change of illumination has in most cases a significant effect on the performance of the momentum-based methods.

To at least give an illustration of how the change in illumination influences the change in the performance of momentum-based methods, for every pair of the 707 illuminations of the CroP dataset generator the corresponding datasets were created and then the per-image difference in reproduction angular errors of the momentum-based methods have been calculated. The only information taken for each pair were the maximum of the per-image differences and the angle between the illuminations used to generate the datasets. The pairs were then grouped by ranges of the angles between their illuminations and for each range the mean of the maximum differences 
was calculated. The results are shown in Figure 6 and Figure 7. It can be seen that the difference of the performance of momentum-based methods raises on average close to linearly with the increase of the angle between the initial and the changed illumination colors.

In short, the larger the difference between the illuminations, the larger also the expected maximum difference in errors obtained by momentum-based methods on the images affected by the illuminations.

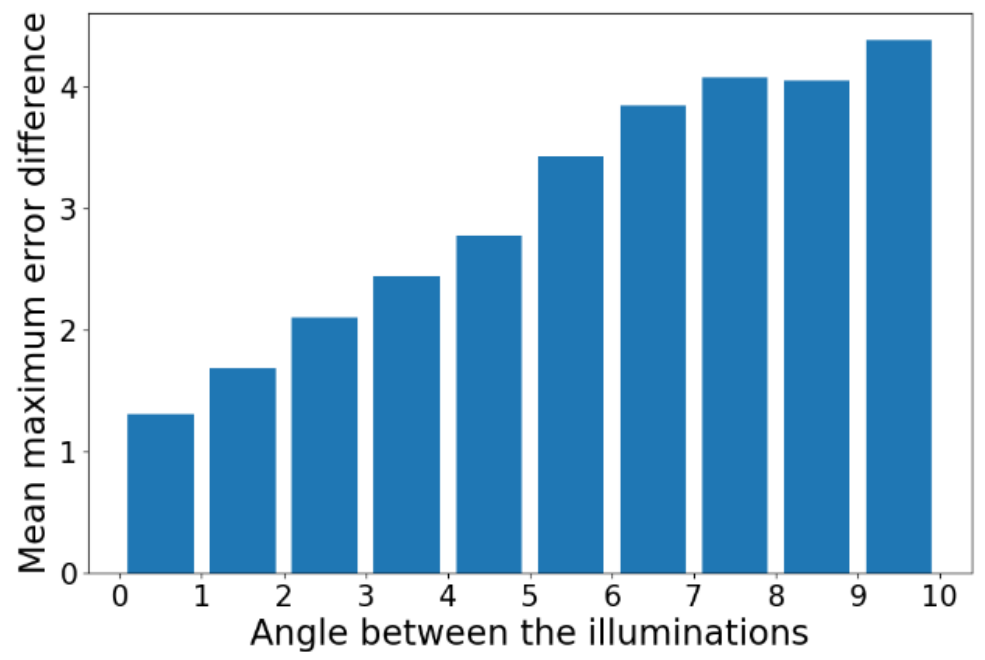

Figure 6. Mean per-image maximum difference of reproduction angular error obtained by the Gray-world method for pairs of image datasets generated by CroP under illuminations grouped by angles between them.

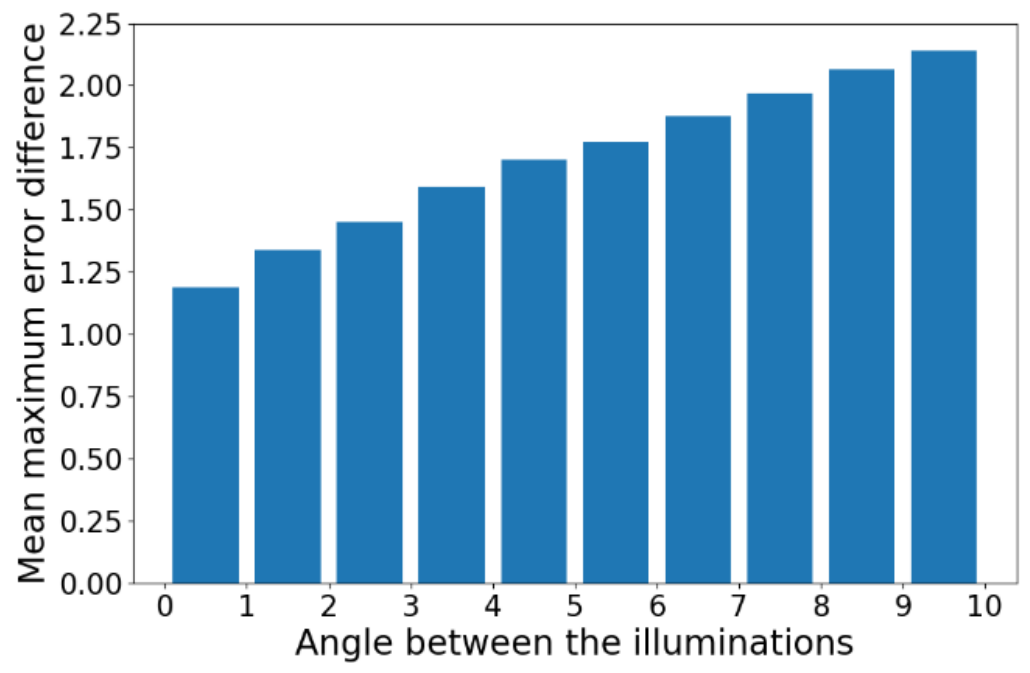

Figure 7. Mean per-image maximum difference of reproduction angular error obtained by the Shades-ofGray method for pairs of image datasets generated by $\mathrm{CroP}$ under illuminations grouped by angles between them.

\section{Conclusions}

In this paper some basic properties that should be expected from the images generated through data augmentation by illumination simulation have been examined. It has been shown how the commonly used data augmentation by illumination simulation has no effect on the performance 
of momentum-based methods. This means that the usual form of augmentation also has practically no use at all when learning the best parameters for momentum-based methods by minimizing the estimation error. On the other hand, real illuminations have such an effect that the performance of momentum-based methods on the same images can on average differ by several degrees depending on the scene illumination. Additionally, in many cases even slight changes from one illumination to another bring significant change in performance of momentum-based methods with the maximum of the expected reproduction angular error obtained on images growing linearly with the angle between the illuminations. Future research will include designing more extensive tests for examining further limits of the described data augmentation and looking for better ways of doing it without relying on generators like CroP. As for using CroP, some future improvements may include its extension to other materials as well as increasing the number of supported illuminations and using various other light sources with different spectral characteristics. Additionally, the effect of using a $3 \times 3$ matrix instead of a diagonal von Kries matrix for the sake of enabling simple data augmentation is also going to be researched.

\section{ACKNOWLEDGEMENTS}

This work was supported by the Croatian Science Foundation under Project IP-06-2016-2092.

\section{REFERENCES}

[1] Ebner, M. (2007). Color constancy (Vol. 7). John Wiley \& Sons.

[2] Barnard, K., Cardei, V., \& Funt, B. (2002). A comparison of computational color constancy algorithms. I: Methodology and experiments with synthesized data. IEEE transactions on Image Processing, 11(9), 972-984.

[3] Land, E. H. (1977). The retinex theory of color vision. Scientific american, 237(6), 108-129.

[4] Funt, B., \& Shi, L. (2010, January). The rehabilitation of maxrgb. In Color and imaging conference (Vol. 2010, No. 1, pp. 256-259). Society for Imaging Science and Technology.

[5] Banić, N., \& Lončarić, S. (2013). Using the random sprays Retinex algorithm for global illumination estimation. arXiv preprint arXiv:1310.0307.

[6] Banić, N., \& Lončarić, S. (2014, August). Color Rabbit: Guiding the distance of local maximums in illumination estimation. In 2014 19th International Conference on Digital Signal Processing (pp. 345350). IEEE.

[7] Banić, N., \& Lončarić, S. (2014, October). Improving the white patch method by subsampling. In 2014 IEEE International Conference on Image Processing (ICIP) (pp. 605-609). IEEE.

[8] Buchsbaum, G. (1980). A spatial processor model for object colour perception. Journal of the Franklin institute, 310(1), 1-26.

[9] Finlayson, G. D., \& Trezzi, E. (2004, January). Shades of gray and colour constancy. In Color and Imaging Conference (Vol. 2004, No. 1, pp. 37-41). Society for Imaging Science and Technology.

[10] Van De Weijer, J., Gevers, T., \& Gijsenij, A. (2007). Edge-based color constancy. IEEE Transactions on image processing, 16(9), 2207-2214.

[11] Cheng, D., Prasad, D. K., \& Brown, M. S. (2014). Illuminant estimation for color constancy: why spatial-domain methods work and the role of the color distribution. JOSA A, 31(5), 1049-1058.

[12] Finlayson, G. D., Hordley, S. D., \& Tastl, I. (2006). Gamut constrained illuminant estimation. International Journal of Computer Vision, 67(1), 93-109.

[13] Van De Weijer, J., Schmid, C., \& Verbeek, J. (2007, October). Using high-level visual information for color constancy. In 2007 IEEE 11th International Conference on Computer Vision (pp. 1-8). IEEE.

[14] Gijsenij, A., \& Gevers, T. (2007, June). Color constancy using natural image statistics. In 2007 IEEE Conference on Computer Vision and Pattern Recognition (pp. 1-8). IEEE.

[15] Gehler, P. V., Rother, C., Blake, A., Minka, T., \& Sharp, T. (2008, June). Bayesian color constancy revisited. In 2008 IEEE Conference on Computer Vision and Pattern Recognition (pp. 1-8). IEEE.

[16] Chakrabarti, A., Hirakawa, K., \& Zickler, T. (2011). Color constancy with spatio-spectral statistics. IEEE Transactions on Pattern Analysis and Machine Intelligence, 34(8), 1509-1519. 
[17] Banić, N., \& Lončarić, S. (2014). Color cat: Remembering colors for illumination estimation. IEEE Signal Processing Letters, 22(6), 651-655.

[18] Banić, N., \& Lončarić, S. (2015, September). Using the red chromaticity for illumination estimation. In 2015 9th International Symposium on Image and Signal Processing and Analysis (ISPA) (pp. 131136). IEEE.

[19] Banic, N., \& Loncaric, S. (2015, March). Color Dog-Guiding the Global Illumination Estimation to Better Accuracy. In VISAPP (1) (pp. 129-135).

[20] Finlayson, G. D. (2013). Corrected-moment illuminant estimation. In Proceedings of the IEEE International Conference on Computer Vision (pp. 1904-1911).

[21] Cheng, D., Price, B., Cohen, S., \& Brown, M. S. (2015). Effective learning-based illuminant estimation using simple features. In Proceedings of the IEEE Conference on Computer Vision and Pattern Recognition (pp. 1000-1008).

[22] Barron, J. T. (2015). Convolutional color constancy. In Proceedings of the IEEE International Conference on Computer Vision (pp. 379-387).

[23] Barron, J. T., \& Tsai, Y. T. (2017). Fast fourier color constancy. In Proceedings of the IEEE Conference on Computer Vision and Pattern Recognition (pp. 886-894).

[24] Bianco, S., Cusano, C., \& Schettini, R. (2015). Color constancy using CNNs. In Proceedings of the IEEE Conference on Computer Vision and Pattern Recognition Workshops (pp. 81-89).

[25] Shi, W., Loy, C. C., \& Tang, X. (2016, October). Deep specialized network for illuminant estimation. In European conference on computer vision (pp. 371-387). Springer, Cham.

[26] Hu, Y., Wang, B., \& Lin, S. (2017). Fc4: Fully convolutional color constancy with confidenceweighted pooling. In Proceedings of the IEEE Conference on Computer Vision and Pattern Recognition (pp. 4085-4094).

[27] Qiu, J., Xu, H., Ma, Y., \& Ye, Z. (2018). PILOT: A Pixel Intensity Driven Illuminant Color Estimation Framework for Color Constancy. arXiv preprint arXiv:1806.09248.

[28] Koščević, K., Banić, N., \& Lončarić, S. (2019). Color beaver: Bounding illumination estimations for higher accuracy. In Proceedings of the 14th International Joint Conference on Computer Vision, Imaging and Computer Graphics Theory and Applications (VISAPP 2019) (p. 183).

[29] Akbarinia, A., \& Parraga, C. A. (2017). Colour constancy beyond the classical receptive field. IEEE transactions on pattern analysis and machine intelligence, 40(9), 2081-2094.

[30] Woo, S. M., Lee, S. H., Yoo, J. S., \& Kim, J. O. (2017). Improving color constancy in an ambient light environment using the phong reflection model. IEEE Transactions on Image Processing, 27(4), 1862-1877.

[31] Yang, K. F., Gao, S. B., \& Li, Y. J. (2015). Efficient illuminant estimation for color constancy using grey pixels. In Proceedings of the IEEE conference on computer vision and pattern recognition (pp. 2254-2263).

[32] Gao, S. B., Yang, K. F., Li, C. Y., \& Li, Y. J. (2015). Color constancy using double-opponency. IEEE transactions on pattern analysis and machine intelligence, 37(10), 1973-1985.

[33] Banić, N., Koščević, K., Subašić, M., \& Lončarić, S. (2019). Crop: Color constancy benchmark dataset generator. arXiv preprint arXiv:1903.12581.

[34] Banic, N., \& Loncaric, S. (2018). Flash and Storm: Fast and Highly Practical Tone Mapping based on Naka-Rushton Equation. In VISIGRAPP (4: VISAPP) (pp. 47-53).

[35] Finlayson, G. D., Zakizadeh, R., \& Gijsenij, A. (2016). The reproduction angular error for evaluating the performance of illuminant estimation algorithms. IEEE transactions on pattern analysis and machine intelligence, 39(7), 1482-1488.

[36] Laakom, F., Raitoharju, J., Iosifidis, A., Nikkanen, J., \& Gabbouj, M. (2019, December). Color constancy convolutional autoencoder. In 2019 IEEE Symposium Series on Computational Intelligence (SSCI) (pp. 1085-1090). IEEE.

[37] Laakom, F., Passalis, N., Raitoharju, J., Nikkanen, J., Tefas, A., Iosifidis, A., \& Gabbouj, M. (2020). Bag of color features for color constancy. IEEE Transactions on Image Processing, 29, 7722-7734.

[38] von Kries, J. (1902). Theoretische studien über die umstimmung des sehorgans. Festschrift der Albrecht-Ludwigs-Universität, 145-158.

[39] Barnard, K., Martin, L., Funt, B., \& Coath, A. (2002). A data set for color research. Color Research \& Application: Endorsed by Inter-Society Color Council, The Colour Group (Great Britain), Canadian Society for Color, Color Science Association of Japan, Dutch Society for the Study of Color, The Swedish Colour Centre Foundation, Colour Society of Australia, Centre Français de la Couleur, 27(3), 147-151. 
[40] Rizzi, A., Gatta, C., \& Marini, D. (2003, January). YACCD: yet another color constancy database. In Color Imaging VIII: Processing, Hardcopy, and Applications (Vol. 5008, pp. 24-35). International Society for Optics and Photonics.

[41] Weber, A. (2019). SIPI Image Database -Mmisc. [Online]. Available: http://sipi.usc.edu/database/database.php?volume=misc

[42] Banić, N., Koščević, K., \& Lončarić, S. (2017). Unsupervised learning for color constancy. arXiv preprint arXiv:1712.00436.

[43] Wilcoxon, F. (1992). Individual comparisons by ranking methods. In Breakthroughs in statistics (pp. 196-202). Springer, New York, NY.

[44] Hordley, S. D., \& Finlayson, G. D. (2004, August). Re-evaluating colour constancy algorithms. In Proceedings of the 17th International Conference on Pattern Recognition, 2004. ICPR 2004. (Vol. 1, pp. 76-79). IEEE.

[45] Rupert Jr, G. (2012). Simultaneous statistical inference. Springer Science \& Business Media.

[46] Abdi, H. (2007). Bonferroni and Šidák corrections for multiple comparisons. Encyclopedia of measurement and statistics, 3, 103-107.

\section{AUTHORS}

Nikola Banić received B.Sc., M.Sc., and Ph.D. degrees in computer science in 2011, 2013, and 2016, respectively. He is currently working as a senior computer vision engineer at Gideon Brothers, Croatia. He has worked in real-time image enhancement for embedded systems, digital signature recognition, people tracking and counting, and image processing for stereo vision. His research interests include image enhancement, color constancy, image processing for stereo vision, and tone mapping.

Karlo Koščević received B.Sc. and M.Sc. degrees in computer science in 2016 and 2018, respectively. He is currently in his second year of the technical sciences in the scientific field of computing Ph.D. program at the Faculty of Electrical Engineering and Computing, University of Zagreb, Croatia. His research interests include image processing, image analysis, and deep learning. His current research is in the area of color constancy with a focus on learning-based methods for illumination estimation.

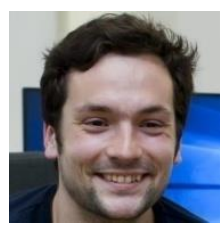

Marko Subašić received a Ph.D. degree from the Faculty of Electrical Engineering and Computing at the University of Zagreb in 2007. Since 1999, he has been working at the Department for Electronic Systems and Information Processing at the Faculty of Electrical Engineering and Computing at the University of Zagreb, currently as an associate professor. He teaches several courses at the graduate and undergraduate levels. His research interests lie in image processing and analysis and neural networks, with a particular interest in image segmentation, detection techniques, and deep learning. He is a

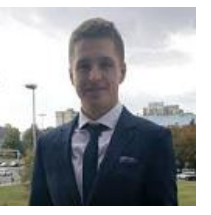
member of the IEEE - Computer Society, the Croatian Center for Computer Vision, the Croatian Society for Biomedical Engineering and Medical Physics, and the Centre of Research Excellence for Data Science and Advanced Cooperative Systems.

Sven Lončarić received B.Sc., M.Sc., and Ph.D. degrees in 1985, 1989, and 1994, respectively. After earning his doctoral degree, he continued his academic career at the Faculty of Electrical Engineering and Computing, University of Zagreb, where he is currently a full professor. He was an assistant professor at the Department of Electrical and Computer Engineering, New Jersey Institute of Technology, NJ, USA, from 20012003. His main areas of research are image processing and analysis. Together with his

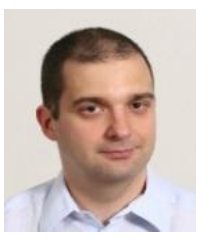
students and collaborators, he has published more than 200 publications in scientific peer-reviewed journals and has presented his work at international conferences. He is a senior member of the IEEE, director of the Center for Artificial Intelligence, and co-director of the national Center of Research Excellence for Data Science and Advanced Cooperative Systems. He is a recipient of several awards for his scientific and professional work.

(C) 2020 By AIRCC Publishing Corporation. This article is published under the Creative Commons Attribution (CC BY) license. 